\title{
Relations for Moments of Dual Generalized Order Statistics from Exponentiated Rayleigh Distribution and Associated Inference
}

\author{
M. A. R. Khan, R. U. Khan*, B. Singh \\ Department of Statistics and Operations Research, Aligarh Muslim University, Aligarh-202 002, India
}

\section{ARTICLE INFO}

Article History

Received 21 Jan 2018

Accepted 25 Sep 2019

\section{Keywords}

Dual generalized order statistics

Order statistics

Lower records

Single moments

Product moments

Recurrence relations

Exponentiated Rayleigh distribution

and characterization

AMS 2010 Subject Classifications

$62 \mathrm{G} 30,62 \mathrm{E} 10,60 \mathrm{E} 05$

\section{ABSTRACT}

In this paper we obtain exact expressions and some recurrence relations satisfied by single and product moments of dual generalized order statistics from exponentiated Rayleigh distribution. These relations are deduced for moments of order statistics and lower record values. Further, conditional expectation, recurrence relations for single moments and truncated moment are used to characterize this distribution.

(C) 2019 The Authors. Published by Atlantis Press SARL. This is an open access article distributed under the CC BY-NC 4.0 license (http://creativecommons.org/licenses/by-nc/4.0/).

\section{INTRODUCTION}

A random variable $X$ is said to have exponentiated Rayleigh distribution [1] if its probability density function $(p d f)$ is of the form

$$
f(x)=2 \alpha \beta x\left(1-e^{-\beta x^{2}}\right)^{\alpha-1} e^{-\beta x}, x>0, \alpha, \beta>0
$$

with distribution function $(d f)$

$$
F(x)=\left(1-e^{-\beta x^{2}}\right)^{\alpha}, x>0, \alpha, \beta>0 .
$$

The exponentiated Rayleigh distribution has many characteristics which are quite common to gamma, Weibull and exponentiated exponential distributions. The exponentiated Rayleigh distribution for the distribution function and the density function are found to have closed forms. Consequently, it can be applied very compatibly even on censored data.

The concept of lower generalized order statistics ( $\lg$ os) was first introduced by Pawlas and Syznal [2] to enable a common approach to descending ordered random variables like reverse order statistics and lower record values. Further, the concept of lower (dual) generalized order statistics (dgos) was extensively studied by Burkschat et al. [3].

Let $X^{*}(r, n, m, k), r=1,2, \ldots, n$, be the $r$-th dgos and their joint $p d f$ is of the from

$$
k\left(\prod_{j=1}^{n-1} \gamma_{j}\right)\left(\prod_{i=1}^{n-1}\left[F\left(x_{i}\right)\right]^{m_{i}} f\left(x_{i}\right)\right)\left[F\left(x_{n}\right)\right]^{k-1} f\left(x_{n}\right)
$$

for $F^{-1}(1)>x_{1} \geq x_{2} \geq \ldots \geq x_{n}>F^{-1}(0)$. 
For the case $m_{i}=m, i=1,2, \ldots, n-1$, the $p d f$ of $r-$ th $\operatorname{dgos} X^{*}(r, n, m, k)$ is given

$$
f_{X^{*}(r, n, m, k)}(x)=\frac{C_{r-1}}{(r-1) !}[F(x)]^{\gamma_{r}-1} f(x) g_{m}^{r-1}(F(x))
$$

and the joint $p d f$ of $X^{*}(r, n, m, k)$ and $X^{*}(s, n, m, k)$, is

$$
\begin{aligned}
f_{X^{*}(r, n, m, k), X^{*}(s, n, m, k)}(x, y)= & \frac{C_{s-1}}{(r-1) !(s-r-1) !}[F(x)]^{m} f(x) g_{m}^{r-1}(F(x)) \\
& \times\left[h_{m}(F(y))-h_{m}(F(x))\right]^{s-r-1} f(y)[F(y)]^{\gamma_{s}-1}, x>y,
\end{aligned}
$$

where

$$
h_{m}(x)= \begin{cases}-\frac{1}{m+1} x^{m+1}, & m \neq-1 \\ -\log x, & m=-1\end{cases}
$$

and

$$
g_{m}(x)=h_{m}(x)-h_{m}(1), x \in(0,1) .
$$

Several authors utilized the concept of $d g o s$ in their work. References may be made to Pawlas and Szynal [2], Khan et al. [4], Ahsanullah [5,6], Mbah and Ahsanullah [7], Khan et al. [8], Khan and Kumar [9,10] and Khan and Khan [11] among others. In this paper, we mainly focus on the study of $d g o s$ arising from the exponentiated Rayleigh distribution.

\section{RELATIONS FOR SINGLE MOMENTS}

Note that for exponentiated Rayleigh distribution $f(x)$ and $F(x)$ satisfy the relation

$$
2 \alpha \beta F(x)=x^{-1}\left(e^{\beta x^{2}}-1\right) f(x) .
$$

The relation in (6) will be used to derive some simple recurrence relations for the moments of $d g o s$ from the exponentiated Rayleigh distribution.

We shall first establish the exact expression for $E\left[X^{* j}(r, n, m, k)\right]$. Using (4), we have, when $m \neq-1$

$$
\begin{aligned}
E\left[X^{* j}(r, n, m, k)\right] & =\frac{C_{r-1}}{(r-1) !} \int_{0}^{\infty} x^{j}[F(x)]^{\gamma_{r}-1} f(x) g_{m}^{r-1}(F(x)) d x \\
& =\frac{C_{r-1}}{(r-1) !} I_{j}\left(\gamma_{r}-1, r-1\right),
\end{aligned}
$$

where

$$
I_{j}(a, b)=\int_{0}^{\infty} x^{j}[F(x)]^{a} f(x) g_{m}^{b}(F(x)) d x
$$

On expanding $g_{m}^{b}(F(x))=\left(\frac{1}{m+1}\left\{1-(F(x))^{m+1}\right\}\right)^{b}$ binomially in (8), we get

$$
I_{j}(a, b)=\frac{1}{(m+1)^{b}} \sum_{u=0}^{b}(-1)^{u}\left(\begin{array}{l}
b \\
u
\end{array}\right) \int_{0}^{\infty} x^{j}[F(x)]^{a+u(m+1)} f(x) d x .
$$

Making the substitution $t=[F(x)]^{1 / \alpha}$ in (9), we find that

$$
I_{j}(a, b)=\frac{\alpha}{\beta^{j / 2}(m+1)^{b}} \sum_{u=0}^{b}(-1)^{u}\left(\begin{array}{l}
b \\
u
\end{array}\right) \int_{0}^{1}[-\ln (1-t)]^{j / 2} t^{\alpha[a+u(m+1)+1]-1} d t .
$$

On using the logarithmic expansion

$$
[-\ln (1-t)]^{j}=\left(\sum_{p=1}^{\infty} \frac{t^{p}}{p}\right)^{j}=\sum_{p=0}^{\infty} z_{p}(j) t^{j+p},|t|<1,
$$


where $z_{p}(j)$ is the coefficient of $t^{j+p}$ in the expansion of $\left(\sum_{p=1}^{\infty} \frac{t^{p}}{p}\right)^{j}$ (see Balakrishnan and Cohen [12], p. 44), we get

$$
\begin{aligned}
I_{j}(a, b) & =\frac{\alpha}{\beta^{j / 2}(m+1)^{b}} \sum_{p=0}^{\infty} \sum_{u=0}^{b}(-1)^{u}\left(\begin{array}{l}
b \\
u
\end{array}\right) z_{p}(j / 2) \int_{0}^{1} t^{\alpha[a+u(m+1)+1]+(j / 2)+p-1} d t \\
& =\frac{1}{\beta^{j / 2}(m+1)^{b}} \sum_{p=0}^{\infty} \sum_{u=0}^{b}(-1)^{u}\left(\begin{array}{l}
b \\
u
\end{array}\right) \frac{z_{p}(j / 2)}{[a+u(m+1)+1+((j / 2)+p) / \alpha]} .
\end{aligned}
$$

When $m=-1$, we have

$$
I_{j}(a, b)=\frac{0}{0}, \text { as } \sum_{u=0}^{b}(-1)^{u}\left(\begin{array}{l}
b \\
u
\end{array}\right)=0 .
$$

Since (12) is of the form $\frac{0}{0}$ at $m \neq 1$, therefore, we have

$$
I_{j}(a, b)=A \sum_{u=0}^{b}(-1)^{u}\left(\begin{array}{l}
b \\
u
\end{array}\right) \frac{[\alpha\{a+u(m+1)+1\}+(j / 2)+p]^{-1}}{(m+1)^{b}},
$$

where

$$
A=\frac{1}{\beta^{j / 2}} \sum_{p=0}^{\infty} z_{p}(j / 2)
$$

Differentiating numerator and denominator of (13) $b$ times with respect to $m$, we get

$$
I_{j}(a, b)=A \sum_{u=0}^{b}(-1)^{u+b}\left(\begin{array}{l}
b \\
u
\end{array}\right) \frac{u^{b}}{[a+u(m+1)+1+((j / 2)+p) / \alpha]^{b+1}}, \quad b>0 .
$$

On applying L' Hospital rule, we have

$$
\lim _{m \rightarrow-1} I_{j}(a, b)=A \sum_{u=0}^{b}(-1)^{u+b}\left(\begin{array}{l}
b \\
u
\end{array}\right) \frac{u^{b}}{[a+1+((j / 2)+p) / \alpha]^{b+1}} .
$$

But for all integers $n \geq 0$ and for all real numbers $x$, we have Ruiz [13]

$$
\sum_{i=0}^{n}(-1)^{i}\left(\begin{array}{c}
n \\
i
\end{array}\right)(x-i)^{n}=n !
$$

Therefore,

$$
\sum_{u=0}^{b}(-1)^{u+b}\left(\begin{array}{l}
b \\
u
\end{array}\right) u^{b}=b !
$$

On substituting (16) in (14), we find that

$$
I_{j}(a, b)=\frac{b !}{\beta^{j / 2}} \sum_{p=0}^{\infty} \frac{z_{p}(j / 2)}{[a+1+((j / 2)+p) / \alpha]^{b+1}}, m=-1 .
$$

Now substituting for $I_{j}\left(\gamma_{r}-1, r-1\right)$ from (12) in (7) and simplifying, we obtain when $m \neq-1$

$$
\begin{aligned}
E\left[X^{* j}(r, n, m, k)\right]= & \frac{C_{r-1}}{(r-1) ! \beta^{j / 2}(m+1)^{r-1}} \sum_{p=0}^{\infty} \sum_{u=0}^{r-1}(-1)^{u}\left(\begin{array}{c}
r-1 \\
u
\end{array}\right) \\
& \times \frac{z_{p}(j / 2)}{\left[\gamma_{r-u}+((j / 2)+p) / \alpha\right]}
\end{aligned}
$$


and when $m=-1$, in view of (17) and (7), we have

$$
E\left[X^{* j}(r, n,-1, k)\right]=E\left[\left(Z_{r}^{(k)}\right)^{j}\right]=\frac{k^{r}}{\beta^{j / 2}} \sum_{p=0}^{\infty} \frac{z_{p}(j / 2)}{[k+((j / 2)+p) / \alpha]^{r}},
$$

where $Z_{r}^{(k)}$ denote the $k$-th lower record value.

Identity 2.1. For $\gamma_{r} \geq 1, k \geq 1,1 \leq r \leq n$ and $m \neq-1$

$$
\sum_{u=0}^{r-1}(-1)^{u}\left(\begin{array}{c}
r-1 \\
u
\end{array}\right) \frac{1}{\gamma_{r-u}}=\frac{(r-1) !(m+1)^{r-1}}{\prod_{t=1}^{r} \gamma_{t}}
$$

Proof. At $j=0$ in (18), we have

$$
1=\frac{C_{r-1}}{(r-1) !(m+1)^{r-1}} \sum_{p=0}^{\infty} \sum_{u=0}^{r-1}(-1)^{u}\left(\begin{array}{c}
r-1 \\
u
\end{array}\right) \frac{z_{p}(0)}{\left[\gamma_{r-u}+(p / \alpha)\right]}
$$

Note that, if $j=0$, then

$z_{p}(0)=1, p=0$ and $z_{p}(0)=0, p>0$ (see Shawky and Bakoban [14]) and hence the result given in (20).

\subsection{Special Cases}

i. Putting $m=0, k=1$ in (18), the explicit formula for single moments of order statistics of the exponentiated Rayleigh distribution can be obtained as

$$
E\left(X_{n-r+1: n}^{j}\right)=\frac{C_{r: n}}{\beta^{j / 2}} \sum_{p=0}^{\infty} \sum_{u=0}^{r-1}(-1)^{u}\left(\begin{array}{c}
r-1 \\
u
\end{array}\right) \frac{z_{p}(j / 2)}{[n-r+1+u+\{(j / 2)+p\} / \alpha]} .
$$

That is

$$
E\left(X_{r: n}^{j}\right)=\frac{C_{r: n}}{\beta^{j / 2}} \sum_{p=0}^{\infty} \sum_{u=0}^{n-r}(-1)^{u}\left(\begin{array}{c}
n-r \\
u
\end{array}\right) \frac{z_{p}(j / 2)}{[r+u+((j / 2)+p) / \alpha]},
$$

where

$$
C_{r: n}=\frac{n !}{(r-1) !(n-r) !}
$$

ii. Putting $k=1$ in (19), we deduce the explicit expression for the moments of lower record values from the exponentiated Rayleigh distribution as

$$
E\left(X_{L(r)}^{j}\right)=\frac{1}{\beta^{j / 2}} \sum_{p=0}^{\infty} \frac{z_{p}(j / 2)}{[1+\{(j / 2)+p\} / \alpha]^{r}} .
$$

Now we obtain the recurrence relations for single moments of exponentiated Rayleigh distribution in the following theorem.

Theorem 2.1. For the distribution as given in (2) for $2 \leq r \leq n, n \geq 2$ and $k=1,2, \ldots$

$$
\begin{aligned}
E\left[X^{* j}(r, n, m, k)\right]= & E\left[X^{* j}(r-1, n, m, k)\right] \\
& +\frac{j}{2 \alpha \beta \gamma_{r}}\left\{E\left[X^{* j-2}(r, n, m, k)\right]-E\left[\varphi\left(X^{*}(r, n, m, k)\right)\right]\right\}
\end{aligned}
$$

where

$$
\varphi(x)=x^{j-2} e^{\beta x^{2}}
$$


Proof. In view of Khan et al. [15], note that

$$
\begin{aligned}
E\left[X^{* j}(r, n, m, k)\right] & -E\left[X^{* j}(r-1, n, m, k)\right] \\
= & -\frac{j C_{r-1}}{\gamma_{r}(r-1) !} \int_{0}^{\infty} x^{j-1}[F(x)]^{\gamma_{r}} g_{m}^{r-1}(F(x)) d x .
\end{aligned}
$$

On using (6) in (22), we get

$$
\begin{aligned}
E\left[X^{* j}(r, n, m, k)\right]-E\left[X^{* j}(r-1, n, m, k)\right]= & \frac{j}{2 \alpha \beta \gamma_{r}}\left\{\frac{C_{r-1}}{(r-1) !} \int_{0}^{\infty} x^{j-2}[F(x)]^{\gamma_{r}-1} f(x) g_{m}^{r-1}(F(x)) d x\right. \\
& \left.-\frac{C_{r-1}}{(r-1) !} \int_{0}^{\infty} x^{j-2} e^{\beta x^{2}}[F(x)]^{\gamma_{r}-1} f(x) g_{m}^{r-1}(F(x)) d x\right\}
\end{aligned}
$$

and hence the result given in (21).

Remark 2.1. Putting $m=0, k=1$, in (21), we obtain a recurrence relation for single moments of order statistics of the exponentiated Rayleigh distribution in the form

$$
E\left(X_{n-r+1: n}^{j}\right)=E\left(X_{n-r+2: n}^{j}\right)+\frac{j}{2 \alpha \beta(n-r+1)}\left\{E\left(X_{n-r+1: n}^{j-2}\right)-E\left(\varphi\left(X_{n-r+1: n}\right)\right)\right\}
$$

Replacing $(n-r+1)$ by $(r-1)$, we have

$$
E\left(X_{r: n}^{j}\right)=E\left(X_{r-1: n}^{j}\right)+\frac{j}{2 \alpha \beta(r-1)}\left\{E\left(X_{r: n}^{j-2}\right)-E\left(\varphi\left(X_{r: n}\right)\right)\right\} .
$$

Remark 2.2. Setting $m=-1$ and $k \geq 1$ in (21), we get a recurrence relation for single moments of lower $k$ record values from exponentiated Rayleigh distribution in the form

$$
E\left[\left(Z_{r}^{(k)}\right)^{j}\right]=E\left[\left(Z_{r-1}^{(k)}\right)^{j}\right]+\frac{j}{2 \alpha \beta k}\left\{E\left[\left(Z_{r}^{(k)}\right)^{j-2}\right]-E\left[\varphi\left(Z_{r}^{(k)}\right)\right]\right\} .
$$

\section{RELATIONS FOR PRODUCT MOMENTS}

The explicit expressions for the product moments of $\operatorname{dgos} X^{* i}(r, n, m, k)$ and $X^{* j}(s, n, m, k), 1 \leq r<s \leq n$, can be obtained when $m \neq-1$ as

$$
\begin{aligned}
E\left[X^{* i}(r, n, m, k) X^{* j}(s, n, m, k)\right] & \\
= & \frac{C_{s-1}}{(r-1) !(s-r-1) !} \int_{0}^{\infty} \int_{0}^{x} x^{i} y^{j}[F(x)]^{m} f(x) g_{m}^{r-1}(F(x)) \\
& \times\left[h_{m}(F(y))-h_{m}(F(x))\right]^{s-r-1}[F(y)]^{\gamma_{s}-1} f(y) d y d x .
\end{aligned}
$$

On expanding $g_{m}^{r-1}(F(x))=\left(\frac{1}{m+1}\left\{1-(F(x))^{m+1}\right\}\right)^{r-1}$ binomially in (23), we get

$$
\begin{aligned}
E\left[X^{* i}(r, n, m, k) X^{* j}(s, n, m, k)\right]= & \frac{C_{s-1}}{(r-1) !(s-r-1) !(m+1)^{r-1}} \\
& \times \sum_{u=0}^{r-1}(-1)^{u}\left(\begin{array}{c}
r-1 \\
u
\end{array}\right) \int_{0}^{\infty} \int_{0}^{x} x^{i} y^{j}[F(x)]^{m+u(m+1)} f(x) \\
& \times\left[h_{m}(F(y))-h_{m}(F(x))\right]^{s-r-1}[F(y)]^{\gamma_{s}-1} f(y) d y d x \\
= & \frac{C_{s-1}}{(r-1) !(s-r-1) !(m+1)^{r-1}} \sum_{u=0}^{r-1}(-1)^{u}\left(\begin{array}{c}
r-1 \\
u
\end{array}\right) \\
& \times I_{i, j}\left(m+u(m+1), s-r-1, \gamma_{s}-1\right),
\end{aligned}
$$


where

$$
\begin{aligned}
I_{i, j}(a, b, c)= & \int_{0}^{\infty} \int_{0}^{x} x^{i} y^{j}[F(x)]^{a} f(x)\left[h_{m}(F(y))-h_{m}(F(x))\right]^{b} \\
& \times[F(y)]^{c} f(y) d y d x .
\end{aligned}
$$

Expanding $\left[h_{m}(F(y))-h_{m}(F(x))\right]^{b}$ binomially in (25) after noting that $h_{m}(F(y))-h_{m}(F(x))=g_{m}(F(y))-g_{m}(F(x))$, we get

$$
I_{i, j}(a, b, c)=\frac{1}{(m+1)^{b}} \sum_{v=0}^{b}(-1)^{v}\left(\begin{array}{l}
b \\
v
\end{array}\right) \int_{0}^{\infty} x^{i}[F(x)]^{a+(b-v)(m+1)} f(x) I(x) d x,
$$

where

$$
I(x)=\int_{0}^{x} y^{j}[F(y)]^{c+v(m+1)} f(y) d y .
$$

By setting $t=[F(y)]^{1 / \alpha}$ in (27) and simplifying on the lines of (12), we find that

$$
I(x)=\frac{1}{\beta^{j / 2}} \sum_{p=0}^{\infty} \frac{z_{p}(j / 2)[F(x)]^{c+v(m+1)+1+((j / 2)+p) / \alpha}}{[c+v(m+1)+1+\{(j / 2)+p\} / \alpha]} .
$$

On substituting the expression of $I(x)$ in (26), we have

$$
\begin{aligned}
I_{i, j}(a, b, c)= & \frac{1}{\beta^{j / 2}(m+1)^{b}} \sum_{p=0}^{\infty} \sum_{v=0}^{b}(-1)^{v}\left(\begin{array}{l}
b \\
v
\end{array}\right) \frac{z_{p}(j / 2)}{[c+v(m+1)+1+\{(j / 2)+p\} / \alpha]} \\
& \times \int_{0}^{\infty} x^{i}[F(x)]^{a+c+b(m+1)+1+\{(j / 2)+p\} / \alpha} f(x) d x .
\end{aligned}
$$

Again by setting $w=[F(x)]^{1 / \alpha}$ in (28) and simplifying the resulting expression, we obtain

$$
\begin{aligned}
I_{i, j}(a, b, c)= & \frac{1}{\beta^{(i+j) / 2}(m+1)^{b}} \sum_{p=0}^{\infty} \sum_{q=0}^{\infty} \sum_{v=0}^{b}(-1)^{v}\left(\begin{array}{l}
b \\
v
\end{array}\right) \frac{z_{p}(j / 2)}{[c+v(m+1)+1+\{(j / 2)+p\} / \alpha]} \\
& \times \frac{z_{q}(i / 2)}{[a+c+b(m+1)+2+\{(i / 2)+(j / 2)+p+q\} / \alpha]}
\end{aligned}
$$

and when $m=-1$ that

$$
I_{i, j}(a, b, c)=\frac{0}{0}, \text { as } \sum_{v=0}^{b}(-1)^{v}\left(\begin{array}{l}
b \\
v
\end{array}\right)=0 .
$$

Therefore, on applying L' Hospital rule and using (16), we find that

$$
\begin{aligned}
\lim _{m \rightarrow-1} I_{i, j}(a, b, c)= & \frac{b !}{\beta^{i+j}} \sum_{p=0}^{\infty} \sum_{q=0}^{\infty} \frac{z_{p}(j / 2)}{[c+1+\{(j / 2)+p\} / \alpha]^{b+1}} \\
& \times \frac{z_{q}(i / 2)}{[a+c+2+\{(i / 2)+(j / 2)+p+q\} / \alpha]} .
\end{aligned}
$$

Now on substituting for $I_{i, j}\left(m+u(m+1), s-r-1, \gamma_{s}-1\right)$ from (29) in (24) and simplifying, we obtain when $m \neq-1$

$$
\begin{aligned}
E\left[X^{* i}(r, n, m, k) X^{* j}(s, n, m, k)\right]= & \frac{C_{s-1}}{(r-1) !(s-r-1) ! \beta^{(i+j) / 2}(m+1)^{s-2}} \\
& \times \sum_{p=0}^{\infty} \sum_{q=0}^{\infty} \sum_{u=0}^{r-1} \sum_{v=0}^{s-r-1}(-1)^{u+v}\left(\begin{array}{c}
r-1 \\
u
\end{array}\right)\left(\begin{array}{c}
s-r-1 \\
v
\end{array}\right) \frac{z_{p}(j / 2)}{\left[\gamma_{s-v}+\{(j / 2)+p\} / \alpha\right]} \\
& \times \frac{z_{q}(i / 2)}{\left[\gamma_{r-u}+\{(i / 2)+(j / 2)+p+q\} / \alpha\right]} .
\end{aligned}
$$


and when $m=-1$, in view of (30) and (25), we have

$$
\begin{aligned}
E\left[X^{* i}(r, n,-1, k) X^{* j}(s, n,-1, k)\right] & =E\left[\left(Z_{r}^{(k)}\right)^{i}\left(Z_{s}^{(k)}\right)^{j}\right] \\
& =\frac{(\alpha k)^{s}}{\beta^{(i+j) / 2}} \sum_{p=0}^{\infty} \sum_{q=0}^{\infty} \frac{z_{p}(j) z_{q}(i)}{[\alpha k+(j / 2)+p]^{s-r}[\alpha k+(i / 2)+(j / 2)+p+q]^{r}} .
\end{aligned}
$$

Identity 3.1. For $\gamma_{r}, \gamma_{s} \geq 1, k \geq 1,1 \leq r<s \leq n$ and $m \neq-1$

$$
\sum_{v=0}^{s-r-1}(-1)^{v}\left(\begin{array}{c}
s-r-1 \\
v
\end{array}\right) \frac{1}{\gamma_{s-v}}=\frac{(s-r-1) !(m+1)^{s-r-1}}{\prod_{t=r+1}^{s} \gamma_{t}} .
$$

Proof. At $i=j=0$ in (31), we have

$$
\begin{aligned}
1= & \frac{C_{s-1}}{(r-1) !(s-r-1) !(m+1)^{s-2}} \sum_{p=0}^{\infty} \sum_{q=0}^{\infty} \sum_{u=0}^{r-1} \sum_{v=0}^{s-r-1}(-1)^{u+v}\left(\begin{array}{c}
r-1 \\
u
\end{array}\right)\left(\begin{array}{c}
s-r-1 \\
v
\end{array}\right) \\
& \times \frac{z_{p}(0) z_{q}(0)}{\left[\gamma_{s-v}+(p / \alpha)\right]\left[\gamma_{r-u}+(p+q) / \alpha\right]} .
\end{aligned}
$$

In view of Shawky and Bakoban [14], for $i=j=0$, note that

$$
\alpha_{p}(0)=1, \alpha_{q}(0)=1, p, q=0 \text { and } \alpha_{p}(0)=0, \alpha_{q}(0)=0, p, q>0 .
$$

Therefore,

$$
\sum_{v=0}^{s-r-1}(-1)^{v}\left(\begin{array}{c}
s-r-1 \\
v
\end{array}\right) \frac{1}{\gamma_{s-v}}=\frac{(r-1) !(s-r-1) !(m+1)^{s-2}}{C_{s-1} \sum_{u=0}^{r-1}(-1)^{u}\left(\begin{array}{c}
r-1 \\
u
\end{array}\right) \frac{1}{\gamma_{r-u}}}
$$

Now on using (20), we get the result given in (33).

At $r=0$, (33) reduce to (20).

Remark 3.1. At $j=0$ in (31), we have

$$
E\left[X^{* i}(r, n, m, k)\right]=\frac{C_{r-1}}{(r-1) ! \beta^{i / 2}(m+1)^{r-1}} \sum_{p=0}^{\infty} \sum_{u=0}^{r-1}(-1)^{u}\left(\begin{array}{c}
r-1 \\
u
\end{array}\right) \frac{z_{p}(i / 2)}{\left[\gamma_{r-u}+\{(i / 2)+p\} / \alpha\right]}
$$

which is the exact expression for single moment as given in (18).

\subsection{Special Cases}

i. Putting $m=0, k=1$ in (31), the explicit formula for the product moments of order statistics of the exponentiated Rayleigh distribution is obtained as

$$
\begin{aligned}
E\left(X_{n-r+1: n}^{i} X_{n-s+1: n}^{j}\right)= & \frac{C_{r, s: n}}{\beta(i+j) / 2} \sum_{p=0}^{\infty} \sum_{q=0}^{\infty} \sum_{u=0}^{r-1} \sum_{v=0}^{s-r-1}(-1)^{u+v}\left(\begin{array}{c}
r-1 \\
u
\end{array}\right)\left(\begin{array}{c}
s-r-1 \\
v
\end{array}\right) \\
& \times \frac{z_{p}(j / 2) z_{q}(i / 2)}{[n-s+1+v+\{(j / 2)+p\} / \alpha][n-r+1+u+\{(i / 2)+(j / 2)+p+q\} / \alpha]} .
\end{aligned}
$$

That is

$$
\begin{aligned}
E\left(X_{r: n}^{i} X_{s: n}^{j}\right)= & \frac{C_{r, s: n}}{\beta^{(i+j) / 2}} \sum_{p=0}^{\infty} \sum_{q=0}^{\infty} \sum_{u=0}^{n-s} \sum_{v=0}^{s-r-1}(-1)^{u+v}\left(\begin{array}{c}
n-s \\
u
\end{array}\right)\left(\begin{array}{c}
s-r-1 \\
v
\end{array}\right) \\
& \times \frac{z_{p}(i / 2) z_{q}(j / 2)}{[r-1+v+\{(j / 2)+p\} / \alpha][s-1+u+\{(i / 2)+(j / 2)+p+q\} / \alpha]},
\end{aligned}
$$


where

$$
C_{r, s: n}=\frac{n !}{(r-1) !(s-r-1) !(n-s) !} .
$$

ii. Putting $k=1$ in (32), the explicit formula for the product moments of lower record values for the exponentiated Rayleigh distribution can be obtained as

$$
E\left[X_{L(r)}^{i} X_{L(s)}^{j}\right]=\frac{\alpha^{s}}{\beta^{(i+j) / 2}} \sum_{p=0}^{\infty} \sum_{q=0}^{\infty} \frac{z_{p}(j) z_{q}(i)}{[\alpha+(j / 2)+p]^{s-r}[\alpha+(i / 2)+(j / 2)+p+q]^{r}} .
$$

Theorem 3.1. For the distribution as given in (2), for $1 \leq r<s \leq n, n \geq 2$ and $k=1,2, \ldots$

$$
\begin{aligned}
E\left[X^{* i}(r, n, m, k) X^{* j}(s, n, m, k)\right]-E\left[X^{* i}(r, n, m, k) X^{* j}(s-1, n, m, k)\right] & \\
= & \frac{j}{2 \alpha \beta \gamma_{s}}\left\{E\left[X^{* i}(r, n, m, k) X^{* j-2}(s, n, m, k)\right]\right. \\
& \left.-E\left[\varphi\left(X^{*}(r, n, m, k) X^{*}(s, n, m, k)\right)\right]\right\},
\end{aligned}
$$

where

$$
\varphi(x, y)=x^{i} y^{j-2} e^{\beta x^{2}}
$$

Proof. In view of Khan et al. [15], note that

$$
\begin{aligned}
E\left[X^{* i}(r, n, m, k) X^{* j}(s, n, m, k)\right]-E\left[X^{* i}(r, n, m, k) X^{* j}(s-1, n, m, k)\right] \\
=-\frac{j C_{s-1}}{\gamma_{s}(r-1) !(s-r-1) !} \int_{0}^{\infty} \int_{0}^{x} x^{i} y^{j-1}[F(x)]^{m} f(x) g_{m}^{r-1}(F(x)) \\
\quad \times\left[h_{m}(F(y))-h_{m}(F(x))\right]^{s-r-1}[F(y)]^{\gamma_{s}} d y d x .
\end{aligned}
$$

On using relation (6) in (35), we get

$$
\begin{aligned}
E & {\left[X^{* i}(r, n, m, k) X^{* j}(s, n, m, k)\right]-E\left[X^{* i}(r, n, m, k) X^{* j}(s-1, n, m, k)\right] } \\
= & -\frac{j C_{s-1}}{2 \alpha \beta \gamma_{s}(r-1) !(s-r-1) !}\left\{\int_{0}^{\infty} \int_{0}^{x} x^{i} y^{j-2} e^{\beta x^{2}}[F(x)]^{m} f(x) g_{m}^{r-1}(F(x))\right. \\
& \times\left[h_{m}(F(y))-h_{m}(F(x))\right]^{s-r-1}[F(y)]^{\gamma_{s}-1} f(y) d y d x \\
& \left.-\int_{0}^{\infty} \int_{0}^{x} x^{i} y^{j-2}[F(x)]^{m} f(x) g_{m}^{r-1}(F(x))\left[h_{m}(F(y))-h_{m}(F(x))\right]^{s-r-1}[F(y)]^{\gamma_{s}-1} f(y) d y d x\right\}
\end{aligned}
$$

and hence the result given in (34).

Remark 3.2. Putting $m=0, k=1$ in (34), we obtain recurrence relations for product moments of order statistics of the exponentiated Rayleigh distribution in the form

$$
\begin{aligned}
E\left(X_{n-r+1: n}^{i} X_{n-s+1: n}^{j}\right)-E\left(X_{n-r+1: n}^{i} X_{n-s+2: n}^{j}\right)= & \frac{j}{2 \alpha \beta(n-s+1)} \\
& \times\left\{E\left(X_{n-r+1: n}^{i} X_{n-s+1: n}^{j-2}\right)-E\left(\varphi\left(X_{n-r+1: n} X_{n-s+1: n}\right)\right)\right\} .
\end{aligned}
$$

That is

$$
E\left(X_{r: n}^{i} X_{s: n}^{j}\right)-E\left(X_{r-1: n}^{i} X_{s: n}^{j}\right)=\frac{i}{2 \alpha \beta(r-1)}\left\{E\left(X_{r: n}^{i-2} X_{s: n}^{j}\right)-E\left(\varphi\left(X_{r: n} X_{s: n}\right)\right)\right\} .
$$


Remark 3.3. Setting $m=-1$ and $k \geq 1$, in (34), we obtain the recurrence relations for product moments of lower $k$ record values from exponentiated Rayleigh distribution in the form

$$
\begin{aligned}
& E\left[\left(Z_{r}^{(k)}\right)^{i}\left(Z_{s}^{(k)}\right)^{j}\right]-E\left[\left(Z_{r}^{(k)}\right)^{i}\left(Z_{s-1}^{(k)}\right)^{j}\right] \\
& \quad=\frac{j}{2 \alpha \beta k}\left\{E\left[\left(Z_{r}^{(k)}\right)^{i}\left(Z_{s}^{(k)}\right)^{j-2}\right]-E\left[\varphi\left(Z_{r}^{(k)}\right)\left(Z_{s}^{(k)}\right)\right]\right\} .
\end{aligned}
$$

Remark 3.4. At $i=0$, Theorem 3.1 reduces to Theorem 2.1.

\section{CHARACTERIZATION BY CONDITIONAL EXPECTATION AND RECURRENCE RELATION}

Let $X^{*}(r, n, m, k), r=1,2, \ldots, n$ be $d g o s$ from a continuous population with $d f F(x)$ and $p d f f(x)$, then the conditional $p d f$ of $X^{*}(s, n, m, k)$ given $X^{*}(r, n, m, k)=x, 1 \leq r<s \leq n$, in view of (4) and (5), is

$$
\begin{aligned}
f_{X^{*}(s, n, m, k) \mid X^{*}(r, n, m, k)}(y \mid x)= & \frac{C_{s-1}}{(s-r-1) ! C_{r-1}}[F(x)]^{m-\gamma_{r}+1} \\
& \times\left[h_{m}(F(y))-h_{m}(F(x))\right]^{s-r-1}[F(y)]^{\gamma_{s}-1} f(y), y<x, m \neq-1 \\
f_{Z_{s}^{(k)} \mid Z_{r}^{(k)}(y \mid x)=} & \frac{k^{s-r}}{(s-r-1) !}[\ln F(x)-\ln F(y)]^{s-r-1} \\
& \quad \times\left(\frac{F(y)}{F(x)}\right)^{k-1} \frac{f(y)}{F(x)} d y, y<x, m=-1 .
\end{aligned}
$$

Theorem 4.1. Let $X$ be a non-negative random variable having an absolutely continuous $d f F(x)$ with $F(0)=0$ and $0<F(x)<1$ for all $x>0$, then

$$
\begin{gathered}
E\left[\xi\left\{X^{*}(s, n, m, k)\right\} \mid X^{*}(l, n, m, k)=x\right]=\frac{1}{\beta} \sum_{p=1}^{\infty} \frac{\left(1-e^{-\beta x^{2}}\right)^{p}}{p} \prod_{j=1}^{s-l}\left(\frac{\gamma_{r+j}}{\gamma_{r+j}+(p / \alpha)}\right), \\
E\left[\xi\left(Z_{s}^{(k)}\right) \mid Z_{l}^{(k)}=x\right]=\frac{1}{\beta} \sum_{p=1}^{\infty}\left(\frac{k}{k+(p / \alpha)}\right)^{s-l}\left(1-e^{-\beta x^{2}}\right)^{p / p}, \\
l=r, r+1, m=-1,
\end{gathered}
$$

where

$$
\xi(y)=y^{2}
$$

if and only if

$$
F(x)=\left(1-e^{-\beta x^{2}}\right)^{\alpha}, x>0, \alpha, \beta>0
$$

Proof. When $m \neq-1$, we have from (36) for $s>r+1$

$$
\begin{aligned}
E[\xi\{X(s, n, m, k)\} \mid X(r, n, m, k)=x]= & \frac{C_{s-1}}{(s-r-1) ! C_{r-1}(m+1)^{s-r-1}} \\
& \times \int_{0}^{x} y^{2}\left[1-\left(\frac{F(y)}{F(x)}\right)^{m+1}\right]^{s-r-1}\left(\frac{F(y)}{F(x)}\right)^{\gamma_{s}-1} \frac{f(y)}{F(x)} d y .
\end{aligned}
$$


By setting $u=\frac{F(y)}{F(x)}=\left(\frac{1-e^{-\beta y^{2}}}{1-e^{-\beta x^{2}}}\right)^{\alpha}$ from (2) in (40), we obtain

$$
\begin{aligned}
E[\xi\{X(s, n, m, k)\} \mid X(r, n, m, k)=x]= & \frac{C_{s-1}}{(s-r-1) ! C_{r-1}(m+1)^{s-r-1}} \\
& \times \frac{1}{\beta} \int_{0}^{1}\left[-\ln \left\{1-\left(1-e^{-\beta x^{2}}\right) u^{1 / \alpha}\right\}\right] u^{\gamma_{s}-1}\left(1-u^{m+1}\right)^{s-r-1} d u \\
= & \frac{C_{s-1}}{(s-r-1) ! C_{r-1}(m+1)^{s-r-1} \beta} \sum_{p=1}^{\infty} \frac{\left(1-e^{-\beta x^{2}}\right)^{p}}{p} \\
& \times \int_{0}^{1} u^{(p / \alpha)+\gamma_{s}-1}\left(1-u^{m+1}\right)^{s-r-1} d u .
\end{aligned}
$$

Again by setting $t=u^{m+1}$ in (41), we get

$$
\begin{aligned}
& E[\xi\{X(s, n, m, k)\} \mid X(r, n, m, k)=x]=\frac{C_{s-1}}{(s-r-1) ! C_{r-1}(m+1)^{s-r} \beta}
\end{aligned}
$$

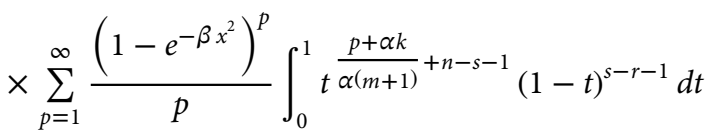

$$
\begin{aligned}
& =\frac{C_{s-1}}{C_{r-1}(m+1)^{s-r} \beta} \sum_{p=1}^{\infty} \frac{\left(1-e^{-\beta x^{2}}\right)^{p}}{p} \frac{\Gamma\left(\frac{p+\alpha k}{\alpha(m+1)}+n-s\right)}{\Gamma\left(\frac{p+\alpha k}{\alpha(m+1)}+n-r\right)} \\
& =\frac{C_{s-1}}{\beta C_{r-1}} \sum_{p=1}^{\infty} \frac{\left(1-e^{-\beta x^{2}}\right)^{p}}{p \prod_{j=1}^{s-r}\left(\gamma_{r+j}+(p / \alpha)\right)},
\end{aligned}
$$

where

$$
\frac{C_{s-1}}{C_{r-1}}=\prod_{j=1}^{s-r} \gamma_{r+j}
$$

and hence the result given in (38).

To prove sufficient part, we have from (36) and (38)

$$
\begin{gathered}
\frac{C_{s-1}}{(s-r-1) ! C_{r-1}(m+1)^{s-r-1}} \int_{0}^{x} y\left[(F(x))^{m+1}-(F(y))^{m+1}\right]^{s-r-1} \\
\times[F(y)]^{\gamma_{s}-1} f(y) d y=[F(x)]^{\gamma_{r+1}} H_{r}(x),
\end{gathered}
$$

where

$$
H_{r}(x)=\frac{1}{\beta} \sum_{p=1}^{\infty} \frac{\left(1-e^{-\beta x^{2}}\right)^{p}}{p} \prod_{j=1}^{s-r}\left(\frac{\gamma_{r+j}}{\gamma_{r+j}+(p / \alpha)}\right) .
$$

Differentiating (42) both sides with respect to $x$, we get

$$
\begin{gathered}
\frac{C_{s-1}[F(x)]^{m} f(x)}{(s-r-2) ! C_{r-1}(m+1)^{s-r-2}} \int_{0}^{x} y\left[(F(x))^{m+1}-(F(y))^{m+1}\right]^{s-r-2}[F(y)]^{\gamma_{s}-1} f(y) d y \\
=H_{r}^{\prime}(x)[F(x)]^{\gamma_{r+1}}+\gamma_{r+1} H_{r}(x)[F(x)]^{\gamma_{r+1}-1} f(x)
\end{gathered}
$$


or

$$
\begin{aligned}
& \gamma_{r+1} H_{r+1}(x)[F(x)]^{\gamma_{r+2}+m} f(x) \\
& \quad=H_{r}^{\prime}(x)[F(x)]^{\gamma_{r+1}}+\gamma_{r+1} H_{r}(x)[F(x)]^{\gamma_{r+1}-1} f(x) .
\end{aligned}
$$

Therefore,

$$
\begin{aligned}
\frac{f(x)}{F(x)} & =\frac{H_{r}^{\prime}(x)}{\gamma_{r+1}\left[H_{r+1}(x)-H_{r}(x)\right]} \\
& =\frac{2 \alpha \beta x e^{-\beta x^{2}}}{1-e^{-\beta x^{2}}},
\end{aligned}
$$

where

$$
\begin{gathered}
H_{r}^{\prime}(x)=2 x e^{-\beta x^{2}} \sum_{p=1}^{\infty}\left(1-e^{-\beta x^{2}}\right)^{p-1} \prod_{j=1}^{s-r}\left(\frac{\gamma_{r+j}}{\gamma_{r+j}+(p / \alpha)}\right), \\
H_{r+1}(x)-H_{r}(x)=\frac{1}{\alpha \beta \gamma_{r+1}} \sum_{p=1}^{\infty}\left(1-e^{-\beta x^{2}}\right)^{p} \prod_{j=1}^{s-r}\left(\frac{\gamma_{r+j}}{\gamma_{r+j}+(p / \alpha)}\right) .
\end{gathered}
$$

Integrating both the sides of (43) with respect to $x$ between $(0, y)$, the sufficiency part is proved.

For the case when $m=-1$, from (37) on using the transformation $u=\frac{F(y)}{F(x)}=\left(\frac{1-e^{-\beta y^{2}}}{1-e^{-\beta x^{2}}}\right)^{\alpha}$, we find that

$$
E\left[\xi\left(Z_{s}^{(k)}\right) \mid Z_{l}^{(k)}=x\right]=A^{*} \int_{0}^{1}(-\ln u)^{s-r-1} u^{k+(p / \alpha)-1} d u,
$$

where

$$
A^{*}=\frac{k^{s-r}}{(s-r-1) ! \beta} \sum_{p=1}^{\infty}\left(1-e^{-\beta x^{2}}\right)^{p} / p
$$

We have Gradshteyn and Ryzhik ([16], p. 551)

$$
\int_{0}^{1}(-\ln x)^{\mu-1} x^{v-1} d x=\frac{\Gamma \mu}{v^{\mu}}, \mu>0, v>0
$$

On using (45) in (44), we have the result given in (39).

Sufficiency part can be proved on the lines of case $m \neq-1$.

Theorem 4.2. Let $X$ be a non-negative random variable having an absolutely continuous $d f F(x)$ with $F(0)=0$ and $0<F(x)<1$ for all $x>0$, then

$$
\begin{aligned}
E\left[X^{* j}(r, n, m, k)\right]= & E\left[X^{* j}(r-1, n, m, k)\right]-\frac{j}{2 \alpha \beta \gamma_{r}} E\left[\varphi\left(X^{*}(r, n, m, k)\right)\right] \\
& +\frac{j}{2 \alpha \beta \gamma_{r}} E\left[X^{* j-2}(r, n, m, k)\right]
\end{aligned}
$$

if and only if

$$
F(x)=\left(1-e^{-\beta x^{2}}\right)^{\alpha}, x>0, \alpha>0, \beta>0
$$


Proof. The necessary part follows immediately from (21). On the other hand if the recurrence relation in (46) is satisfied, then on using (4), we have

$$
\begin{aligned}
& \frac{C_{r-1}}{(r-1) !} \int_{0}^{\infty} x^{j}[F(x)]^{\gamma_{r}-1} f(x) g_{m}^{r-1}(F(x)) d x \\
& =\frac{(r-1) C_{r-1}}{\gamma_{r}(r-1) !} \int_{0}^{\infty} x^{j}[F(x)]^{\gamma_{r}+m} f(x) g_{m}^{r-2}(F(x)) d x \\
& \quad-\frac{j C_{r-1}}{2 \alpha \beta \gamma_{r}(r-1) !} \int_{0}^{\infty} x^{j-2} e^{\beta x^{2}}[F(x)]^{\gamma_{r}-1} f(x) g_{m}^{r-1}(F(x)) d x \\
& \quad+\frac{j C_{r-1}}{2 \alpha \beta \gamma_{r}(r-1) !} \int_{0}^{\infty} x^{j-2}[F(x)]^{\gamma_{r}-1} f(x) g_{m}^{r-1}(F(x)) d x .
\end{aligned}
$$

Integrating the first integral on the right hand side in (47) by parts and simplifying the resulting expression, we get

$$
\begin{aligned}
& \frac{j C_{r-1}}{\gamma_{r}(r-1) !} \int_{0}^{\infty} x^{j-1}[F(x)]^{\gamma_{r}-1} g_{m}^{r-1}(F(x)) d x \\
& \quad \times\left\{F(x)-\frac{1}{2 \alpha \beta x} e^{\beta x^{2}} f(x)+\frac{1}{2 \alpha \beta x} f(x)\right\} d x=0 .
\end{aligned}
$$

Now applying a generalization of the Müntz-Szász Theorem [17] to (48), we get

$$
\frac{f(x)}{F(x)}=\frac{2 \alpha \beta x}{\left(e^{\beta x^{2}}-1\right)},
$$

which proves that

$$
F(x)=\left(1-e^{-\beta x^{2}}\right)^{\alpha}, x>0, \alpha, \beta>0
$$

\section{CHARACTERIZATION BY TRUNCATED MOMENT}

Theorem 5.1. Suppose an absolutely continuous (with respect to Lebesgue measure) random variable $X$ has the $d f F(x)$ and $p d f f(x)$ for $0<x<\infty$, such that $f^{\prime}(x)$ and $E(X \mid X \leq x)$ exist for all $x, 0<x<\infty$, then

$$
E(X \mid X \leq x)=g(x) \eta(x)
$$

where

$$
\eta(x)=\frac{f(x)}{F(x)}
$$

and

$$
g(x)=\frac{1-e^{-\beta x^{2}}}{2 \alpha \beta x e^{-\beta x^{2}}}-\frac{\int_{0}^{x}\left(1-e^{-\beta u^{2}}\right)^{\alpha} d u}{2 \alpha \beta x\left(1-e^{-\beta x^{2}}\right)^{\alpha-1} e^{-\beta x^{2}}},
$$

if and only if

$$
f(x)=2 \alpha \beta x\left(1-e^{-\beta x^{2}}\right)^{\alpha-1} e^{-\beta x^{2}}, x>0, \alpha, \beta>0 .
$$

Proof. In view of Ahsanullah et al. [18] and (1), we have

$$
E(X \mid X \leq x)=\frac{2 \alpha \beta}{F(x)} \int_{0}^{x} u^{2}\left(1-e^{-\beta u^{2}}\right)^{\alpha-1} e^{-\beta x^{2}} d u .
$$


Integrating (50) by parts treating ' $u\left(1-e^{-\beta u^{2}}\right)^{\alpha-1} e^{-\beta x^{2} \prime}$ for integration and rest of the integrant for differentiation, we get

$$
E(X \mid X \leq x)=\frac{1}{F(x)}\left\{x\left(1-e^{-\beta x^{2}}\right)-\int_{0}^{x}\left(1-e^{-\beta u^{2}}\right)^{\alpha} d u\right\} .
$$

After multiplying and dividing by $f(x)$ in (51), we have the result given (49).

To prove sufficient part, we have from (49)

$$
\begin{aligned}
& \quad \frac{1}{F(x)} \int_{0}^{x} u f(u) d u=\frac{g(x) f(x)}{F(x)} \\
& \text { or } \int_{0}^{x} u f(u) d u=g(x) f(x) .
\end{aligned}
$$

Differentiating (52) on both the sides with respect to $x$, we find that

$$
x f(x)=g^{\prime}(x) f(x)+g(x) f^{\prime}(x) .
$$

Therefore,

$$
\begin{aligned}
\frac{f^{\prime}(x)}{f(x)} & =\frac{x-g^{\prime}(x)}{g(x)} \\
& =\frac{2(\alpha-1) \beta x e^{-\beta x^{2}}}{1-e^{-\beta x^{2}}}+\frac{1}{x}-2 \beta x,
\end{aligned}
$$

where

$$
g^{\prime}(x)=x+g(x)\left(\frac{2(\alpha-1) \beta x e^{-\beta x^{2}}}{1-e^{-\beta x^{2}}}+\frac{1}{x}-2 \beta x\right) .
$$

Integrating both the sides in (53) with respect to $x$, we get

$$
f(x)=c x\left(1-e^{-\beta x^{2}}\right)^{\alpha-1} e^{-\beta x^{2}} .
$$

It is known that

$$
\int_{0}^{\infty} f(x) d x=1
$$

Thus,

$$
\frac{1}{c}=\int_{0}^{\infty} x\left(1-e^{-\beta x^{2}}\right)^{\alpha-1} e^{-\beta x^{2}} d x=\frac{1}{2 \alpha \beta},
$$

which proved that

$$
f(x)=2 \alpha \beta x\left(1-e^{-\beta x^{2}}\right)^{\alpha-1} e^{-\beta x^{2}}, x>0, \alpha, \beta>0
$$

\section{CONFLICT OF INTEREST}

The authors are declare no competing interests.

\section{AUTHORS' CONTRIBUTIONS}

The author carried out the proof of the main results and approved the final manuscript.

\section{ACKNOWLEDGMENT}

The authors acknowledge with thanks to both the referee and the Editor-in-Chief Prof. M. Ahsanullah for their fruitful suggestions and comments which led the overall improvement in the manuscript. Authors are also thankful to Prof. A. H. Khan, Aligarh Muslim University, Aligarh, who helped in preparation of this manuscript. 


\section{REFERENCES}

1. M.A.W. Mahmoud, M.G.M. Ghazal, J. Egypt. Math. Soc. 25 (2017), 71-78.

2. P. Pawlas, D. Szynal, Demonstr. Math. XXXIV (2001), 353-358.

3. M. Burkschat, E. Cramer, U. Kamps, Metron. LXI (2003), 13-26. ftp://metron.sta.uniroma1.it/RePEc/articoli/2003-1-13-26.pdf

4. A.H. Khan, R.U. Khan, M. Yaqub, J. Appl. Probab. Stat. 1 (2006), 115-131.

5. M. Ahsanullah, Comm. Statist. Theory Methods. 33 (2004), 2921-2928.

6. M. Ahsanullah, Stat. Methods. 7 (2005), 16-28.

7. A.K. Mbah, M. Ahsanullah, Pak. J. Stat. 23 (2007), 139-146.

8. A.H. Khan, Z. Anwar, S. Chisti, Pak. J. Stat. 26 (2010), 615-628.

9. R.U. Khan, D. Kumar, Appl. Math. Sci. (Ruse). 4 (2010), 2711-2722. http://www.m-hikari.com/ams/ams-2010/ams-53-56-2010/khanAMS5356-2010.pdf

10. R.U. Khan, D. Kumar, Math. Methods Stat. 20 (2011), 150-157.

11. R.U. Khan, M.A. Khan, J. King Saud Univ. Sci. 27 (2015), 285-291.

12. N. Balakrishnan, A.C. Cohen, Order Statistics and Inference: Estimation Methods, Academic Press, San Diego, 1991.

13. S.M. Ruiz, Math. Gaz. 80 (1996), 579-582.

14. A.I. Shawky, R.A. Bakoban, J. Stat. Theory Appl. 7 (2008), 263-277. https://www.kau.edu.sa/files/0008606/researches/24613_33404.pdf

15. R.U. Khan, Z. Anwar, H. Athar, Aligarh J. Stat. 28 (2008), 37-45.

16. I.S. Gradshteyn, I.M. Ryzhik, Table of Integrals, Series, and Products, Academic Press, New York, 2007. https://www.sciencedirect.com/science/article/pii/B9780080471112500030?via\%3Dihu

17. J.S. Hwang, G.D. Lin, Proc. Amer. Math. Soc. 91 (1984), 577-580.

18. M. Ahsanullah, M. Shakil, B.M. Golam Kibria, J. Mod. Appl. Stat. Method. 15 (2016), 316-331. 